\title{
Evaluation De La Qualité Des Noix Brutes D’anacarde En Casamance (Sénégal)
}

\author{
Seydou Ndiaye, \\ (Laboratoire d'Agroforesterie et d'Ecologie), \\ Université Assane Seck de Ziguinchor \\ Mohamed Mahamoud Charahabil, \\ (Maître de Conférences, PhD, Botaniste), \\ Université Assane Seck de Ziguinchor \\ Malaïny Diatta,
}

(Directeur de recherche, $\mathrm{PhD}$, Agroforestier), Institut Sénégalais de

Recherche Agricole/Centre National de Recherches Forestières

\section{Résumé}

La qualité des noix brutes de cajou appréciée à travers le out-turn ou KOR (Kernel Output Ratio) est une donnée très déterminante dans le commerce international de la filière anacarde. Cette étude a pour objectif d'analyser la qualité des noix produites en Casamance, zone la plus productive du Sénégal mais dont les données sur la qualité des noix sont très peu connues ou disparates. L'étude a été réalisée sur un lot de 231 échantillons de $1,5 \mathrm{~kg}$ chacun collectés dans 77 localités sur l'ensemble des trois régions de la Casamance naturelle (Ziguinchor, Sédhiou et Kolda). Sur chaque échantillon, le grainage, le taux de défaut et le KOR ont été calculés. Les résultats de l'étude ont montré que le KOR le plus élevé a été enregistré dans la région de Ziguinchor. Elle varie entre 50-51 lbs avec un taux de défaut moyen de 7,9-9 $\%$ et un grainage de 204-214 noix/Kg. Dans la région Sédhiou, le KOR varie de 49 à $50 \mathrm{lbs}$ avec un taux de défaut de 7,5-10,5\% et un grainage de 210-215 noix $/ \mathrm{Kg}$. La plus faible qualité des noix a été enregistrée à Kolda où le KOR varie entre 46-49 lbs avec un taux de défaut de 9,4-10,6 \% et grainage de 214232 noix/Kg. Ces données montrent que la qualité des noix de la Casamance, notamment à Ziguinchor et à Sédhiou où les valeurs s'approchent des normes de l'UEMOA ( $>$ ou $=50 \mathrm{lbs})$. Toutefois des efforts doivent être menés dans la récolte, le conditionnement et le stockage des noix notamment dans la région de Kolda où le taux de défaut est relativement élevé.

Mots clés : Anacarde, KOR, Taux de défaut, Grainage, Casamance 


\title{
Evaluation of the Quality of Raw Cashew Nuts in Casamance (Senegal)
}

\author{
Seydou Ndiaye, \\ (Laboratoire d'Agroforesterie et d'Ecologie), \\ Université Assane Seck de Ziguinchor \\ Mohamed Mahamoud Charahabil, \\ (Maître de Conférences, $\mathrm{PhD}$, Botaniste), \\ Université Assane Seck de Ziguinchor
}

Malaïny Diatta,

(Directeur de recherche, $\mathrm{PhD}$, Agroforestier), Institut Sénégalais de

Recherche Agricole/Centre National de Recherches Forestières

\begin{abstract}
The quality of the raw cashew nuts appreciated through the out-turn or KOR (Kernel Output Ratio) is a very determining factor in the international trade of the cashew nut sector. The objective of this study is to analyse the quality of nuts produced in Casamance, the most productive zone in Senegal, but for which data on nut quality are very little known or disparate. The study was carried out on a batch of 231 samples of $1.5 \mathrm{~kg}$ each collected in 77 localities throughout the three regions of natural Casamance (Ziguinchor, Sédhiou and Kolda). On each sample, the grading, defect rate and KOR were calculated. The results of the study showed that the highest KOR was recorded in the Ziguinchor region. It varies between 50-51 Ibs with an average defect rate of $7.9-9 \%$ and a kernel of $204-214$ nuts $/ \mathrm{Kg}$. In the Sédhiou region, the KOR varies from 49 to 50 Ibs with a defect rate of $7.5-10.5 \%$ and a kernel weight of $210-215$ nuts $/ \mathrm{Kg}$. The lowest quality of the nuts was recorded in Kolda where the KOR varies between 46-49 Ibs with a defect rate of 9.4$10.6 \%$ and a kernel weight of $214-232$ nuts $/ \mathrm{Kg}$. These data show that the quality of Casamance nuts, especially in Ziguinchor and Sédhiou where the values are close to UEMOA standards ( $>$ or $=50 \mathrm{Ibs}$ ). However, efforts must be made in the harvesting, packaging and storage of nuts, especially in the Kolda region where the defect rate is relatively high.
\end{abstract}

Keywords: Cashew nut, KOR, Defect rate, Grainage, Casamance 


\section{Introduction}

La production mondiale de noix brute est estimée à 4087563 tonnes en 2016 (FAOSTAT, 2019) avec un rendement moyen de 1,3 T/ha (Seters et Konnon, 2018). L'Afrique produit plus de la moitié de la production mondiale (iCA, 2014). La productivité de l'anacarde et la qualité des noix brutes sont des critères de taille dans la sélection semencière. En effet, le net-compte et le KOR permettent d'avoir une idée sur la qualité des noix d'anacarde (DDP, 2002 ; Ba, 2018). Le défi actuel de la filière anacarde est essentiellement lié à la productivité, à la qualité des noix brutes, au délai de livraison des produits, aux réseaux commerciaux et financiers et à la traçabilité des produits qui conditionnent la dynamique des prix d'achat au niveau mondial (Hassine et Matoussi, 2006). Cette situation n'épargne pas les pays producteurs d'anacarde de l'Afrique. Les noix de cajou brutes de l'Inde et du Vietnam se négocient dans l'ordre 1450-1500 US\$ alors que ceux de l'Afrique de l'Ouest sont de l'ordre de 1100-1300 US\$ reportés en fonction de l'origine et de la qualité des noix (Sampat, 2015).

Au Sénégal, la production moyenne est estimée à 28900 tonnes en 2018 (Hien, 2019) dont 97 \% sont destinées à l'exportation (PADEC, 2016). Depuis ces deux dernières décennies, le prix du $\mathrm{kg}$ de noix de cajou brute ne cessent d'augmenter passant de 150 FCFA en 1990 à 1200 FCFA en 2017 (Sène, 2019). Cette valorisation des prix est liée à la forte consommation de l'amande qui fait l'objet d'intense commerce dans le monde (Aina, 1996 ; Gagnon, 1998).

La qualité des noix bruites notamment le KOR demeure cependant le seul baromètre d'appréciation de la qualité des noix produites en Casamance conditionnant ainsi leur prix d'achat. Or les données sur la qualité de ces noix ainsi que leur traçabilité sont quasiment inexistant au Sénégal. C'est pourquoi cette étude se propose de faire la cartographie de la qualité de noix de cajou brute de la région naturelle de la Casamance (Ziguinchor, Sédhiou et Kolda). Cette étude, une des premières, sur cette question dans cette zone qui est la zone à forte production d'anacarde du pays devraient contribuer à une meilleure valorisation de la noix de cajou du Sénégal de manière générale et de la Casamance en particulier.

\section{Materiel Et Methodes}

\section{Sites de collecte des échantillons de noix}

Depuis 2008, la Casamance est subdivisée en trois régions administratives (Figure 1) que sont Ziguinchor, Sédhiou et Kolda. La région de Ziguinchor englobe les départements de Ziguinchor, Oussouye et Bignona. Celle de Sédhiou comprend les départements de Sédhiou, Goudomp et Bounkiling. Et enfin la région de Kolda comprend les départements de Kolda, Vélingara et Médina Yoro Foula. L'ensemble de ces trois régions couvrent 
une superficie de $28464 \mathrm{~km}^{2}$ avec une population de 1664600 habitants (ANSD, 2015). La région de Ziguinchor se situe dans la zone sub-guinéenne côtière (Sagna, 2007) avec une pluviométrie moyenne de $1316 \mathrm{~mm}$ (Camara, 2018). Les régions de Sédhiou et Kolda sont situées dans la zone climatique sub-guinéenne continentale (Sagna, 2007) avec des pluviométries moyennes respectives de $1200 \mathrm{~mm}$ (Ndiaye et al, 2017) et $853 \mathrm{~mm}$ (ANSD, 2011). Au niveau de ces trois régions, les activités socioéconomiques sont dominées par l'agriculture pluviale qui occupe $80 \%$ des ménages (Gaye et al, 2015). Toutefois la culture de l'anacarde est pratiquée par $20 \%$ de la population en Casamance (PADEC, 2016).

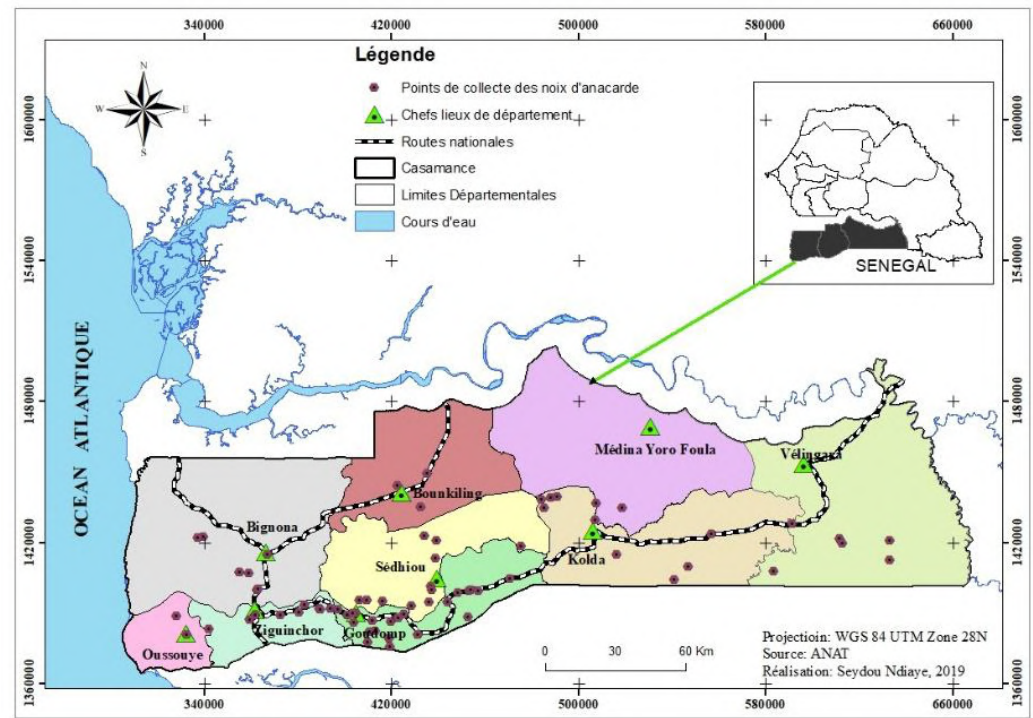

Figure 1 : Carte de localisation des points de collecte des noix brutes d'anacarde

\section{Matériel}

Ces échantillons ont porté sur l'ensemble des variétés de noix rencontrées dans ces trois régions (Figure 2). 

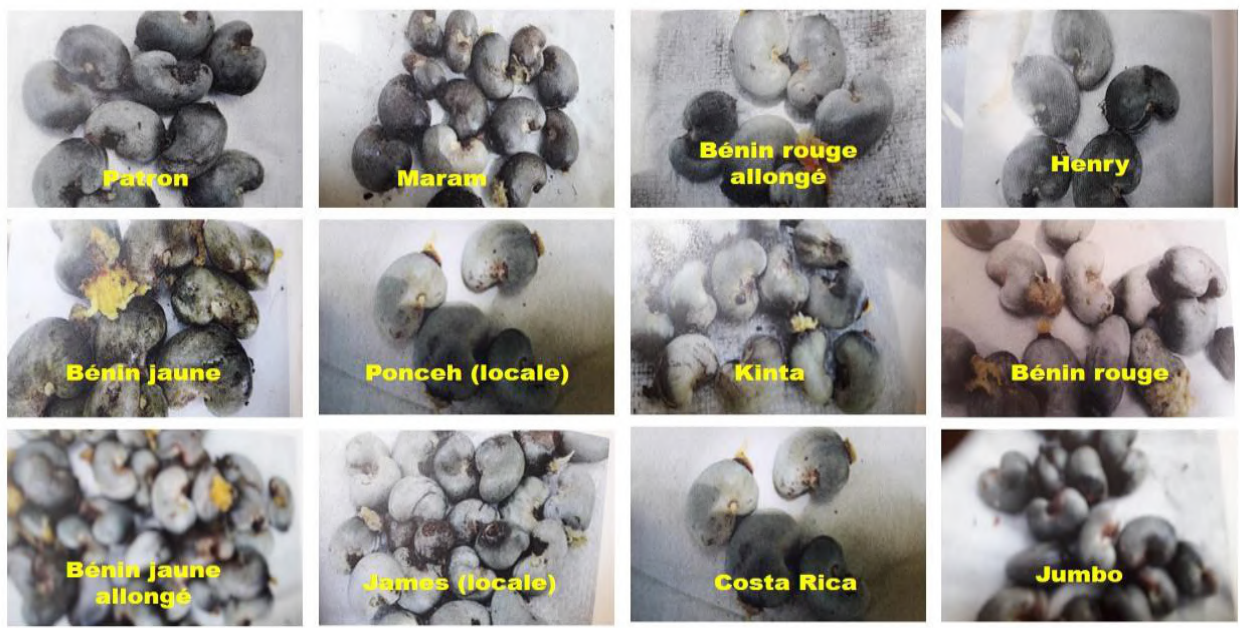

Figure 2 : Diversité des noix rentrées en Casamance (Crédit photo : D. Diallo, ISRA, 2013)

\section{Méthodes}

Les noix ont été collectées au niveau de ces trois régions (Ziguinchor, Sédhiou et Kolda). Au total, 77 villages ont été sélectionnés soient 22 villages dans la région de Ziguinchor, 41 villages dans la région de Sédhiou et 14 villages dans la région de Kolda. L'intensité de la production de noix a été le critère déterminant sur la taille de l'échantillon dans chaque région (Ndiaye, 2014). Au niveau de chaque village, trois (3) échantillons de noix brutes ont été choisis au hasard en raison de $1,5 \mathrm{Kg} /$ échantillon. $\mathrm{Au}$ total, 231 échantillons ont été collectés soit $346,5 \mathrm{Kg}$ de noix. Les échantillons ont été séchés au soleil pendant 5 jours afin de ramener le taux d'humidité entre 5 et $10 \%$.

\section{Paramètres d'analyse et grilles d'appréciation de la qualité des noix Le grainage ou Net-compte}

Le grainage est le nombre de noix par kilogramme. Ce premier critère visible d'appréciation des noix brutes de cajou exprime le nombre graine dans un kilogramme de noix brute. Généralement, la valeur commerciale augmente avec la taille de la noix. Dans ce travail, la grille proposée par (Rongead, 2015) a été utilisée pour évaluer le grainage (Tableau 1). Ainsi dans chaque échantillon de noix, $1 \mathrm{Kg}$ de noix a été pris et le nombre noix a été compté. 
Tableau 1 : Grille d'appréciation du grainage (Rongead, 2015)

\begin{tabular}{ccc}
\hline Classes d'amplitude & $\begin{array}{c}\text { Appréciation de la } \\
\text { qualité }\end{array}$ & Description \\
\hline$<180$ noix & Excellent & Très grosses noix, qualité recherchée \\
{$[180-190[$} & Très bon & Grosses noix, appréciées par les industrielles, bon KOR \\
{$[190-200[$} & Bon & Grosses noix, appréciées par les transformateurs \\
{$[200-210[$} & Moyen & Noix moyennes, plus courant en Afrique de l'Ouest \\
{$[210-220[$} & Très moyen & Noix moyennes, plus courant en Afrique de l'Ouest \\
{$[220-230]$} & Juste acceptable & Petites noix peu recherchées \\
$>230$ & Mauvais & Petites noix, difficiles à transformer \\
\hline
\end{tabular}

\section{Le taux de défaut}

Le taux de défaut traduit la proportion de noix défectueuses (moisies, pourries, immatures) dans l'échantillon (Figure 2). Généralement dans le circuit commerciale, si le taux de défaut est supérieur à $15 \%$, le lot est refusé par les acheteurs et le vendeur se trouve obligé de trier son stock pour pouvoir le vendre (Dandjinou, 2016). Toutes les noix contenues dans chaque kilogramme ont été cassé. Les noix saines (coques et amandes) sont mis dans une cuvette verte en plastique, les noix acceptées à $50 \%$ (coques et amandes) sont mis dans cuvette bleu en plastique et les noix pourries ou immatures (coques et amandes) sont mis dans une cuvette rouge en plastique. Le taux de défaut a été calculé à l'aide de la formule suivante :

Taux de défaut $=\frac{P_{3}+P_{5}}{P_{1}} * 100 \quad($ Rongead, 2015), avec

- $\quad \mathrm{P} 1=$ Poids total de l'échantillon

- $\quad \mathrm{P} 3$ = Poids des amandes + coques rejetées à $50 \%$

- $\mathrm{P} 5=$ Poids des amandes +coques rejetées à $100 \%$ 


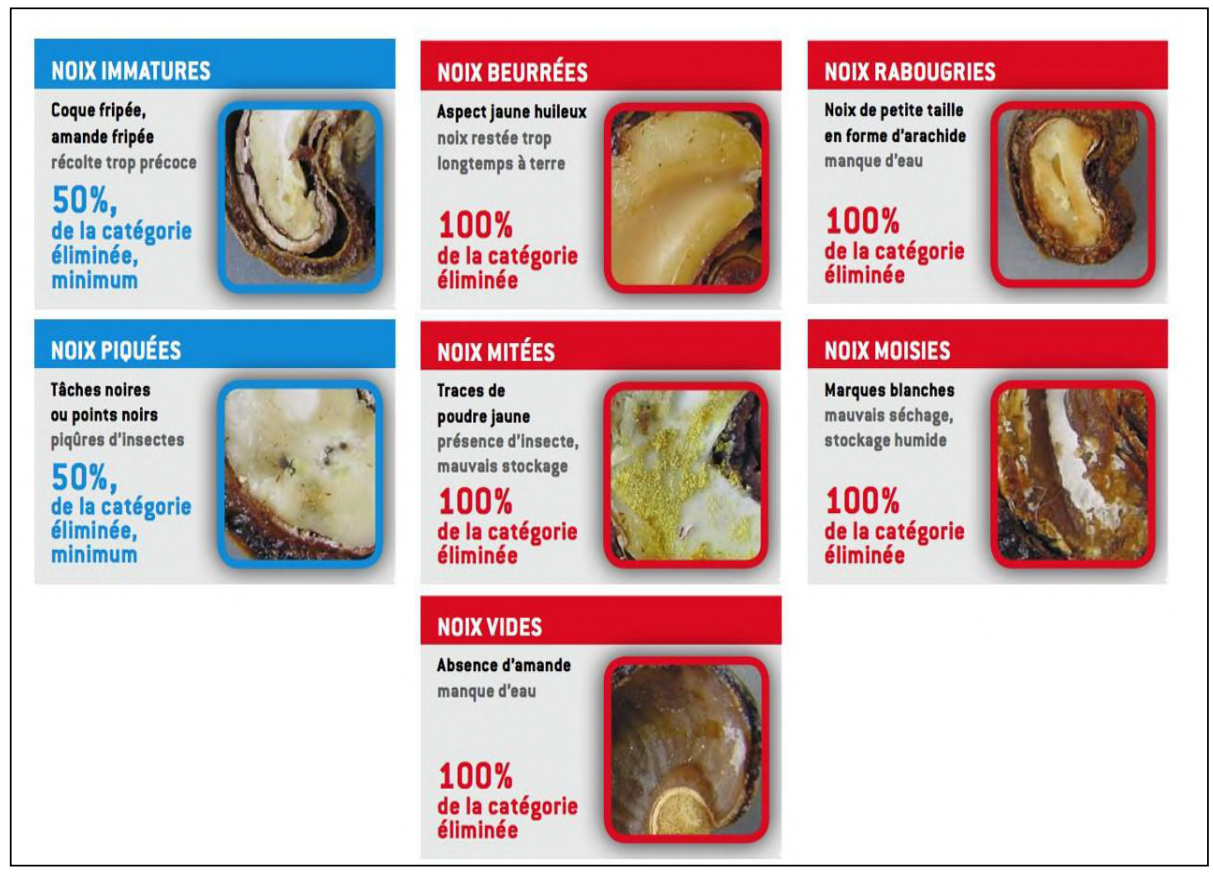

Figure 3 : Critères de classification des noix dans l'analyse du contrôle qualité (Rongead, 2015)

\section{Le rendement en amande ou KOR (Kernel Ouput Ratio)}

C'est l'indicateur le plus important de la qualité des noix brutes. Il permet d'estimer à partir d'un échantillon, la qualité moyenne d'amandes utiles. Il est exprimé en livres $(0,45359 \mathrm{~kg})$ d'amandes par sac de $80 \mathrm{~kg}$ de noix brutes. Ainsi toutes les noix contenues dans chaque échantillon de $1 \mathrm{Kg}$ ont été cassées et classées en trois catégories :

- lot 1 : les noix saines, acceptées à $100 \%$

- lot 2 : les noix piquées, acceptées à $50 \%$

- lot 3 : les noix pourries ou immatures rejetées à $100 \%$

Dans chaque lot, la coque, les pellicules et l'amande ont été séparées et le KOR a été calculé à l'aide de la formule suivant :

$$
\begin{aligned}
& \mathbf{K O R}=\frac{\left(\mathrm{P}_{2}+\left(\frac{\mathrm{P}_{4}}{2}\right)\right) / \mathrm{P}_{1} * 100}{100 * 80 *\left(\frac{1}{0,45359}\right)} \quad \text { (Rongead, 2015) } \mathrm{Où} \\
& \bullet \mathrm{P} 2=\text { Poids des amandes saines (amandes + pellicules) acceptées à } \\
& 100 \% \\
& \bullet \mathrm{P} 4=\text { le poids des amandes }+ \text { pellicules des noix rejetées à } 50 \% \\
& \bullet \mathrm{P} 1=\text { le poids total de l'échantillon de noix }
\end{aligned}
$$

Le rendement en amande et la quantité d'amande utile sont donnés par les formules suivantes : 
- $\quad$ Amande Utile $(\mathrm{AU})=\mathrm{P}_{2}+\frac{P_{4}}{2}$

- $\quad$ Rendement en amande $(\mathrm{Ra})=\frac{\mathrm{P}_{2}+\frac{\mathrm{P}_{4}}{2}}{\mathrm{P}_{1} * 100}$

Le rendement est apprécié à l'aide des critères suivants (Tableau 2)

Tableau 2 : Critère d'appréciation du KOR (Rongead, 2015)

\begin{tabular}{ll}
\hline Catégorie de KOR (Ibs) & Appréciation \\
\hline $\mathbf{4} \mathbf{4 2}$ & Qualité extrêmement mauvaise, très dur à transformer \\
\hline$[\mathbf{4 2 - 4 4}[$ & Très mauvaise qualité, dur à transformer \\
\hline$[\mathbf{4 4 - 4 6}[$ & Mauvaise qualité, peu intéressant pour les transformateurs \\
\hline$[\mathbf{4 6 - 4 8}[$ & Qualité acceptable \\
\hline$[\mathbf{4 8 - 5 0}[$ & Bonne qualité \\
\hline$[\mathbf{5 0 - 5 2}[$ & Très bonne qualité \\
\hline$[\mathbf{5 2 - 5 4}[$ & Excellente qualité \\
\hline$[\mathbf{5 4 - 5 6}[$ & Super qualité, rare et très recherchée \\
\hline
\end{tabular}

\section{Traitement des données}

Les données ont été traitées à l'aide du logiciel R 3.4.2 (Core-team, 2018). Le test de Shapiro (Rakotomala, 2011) a été appliqué à l'ensemble des variables étudiées afin de vérifier les conditions de normalité des données. Ainsi pour le test de comparaison des moyennes, des tests paramétriques (pour les données dont le $\mathrm{p}$-value $<0,05$ ) et non paramétriques ( $\mathrm{p}$-value $>0,05$ ) ont été utilisés. A cela s'ajoute une analyse de variance et le test de Newman Keuls (Shaffer, 2007) pour vérifier l'existence de différences significatives entre les moyennes des différentes provenances de noix (au seuil de $5 \%$ de probabilité) 


\section{Resultats}

\section{Analyse du grainage ou net-compte dans les régions de Ziguinchor, Sédhiou et Kolda}

Les résultats du grainage sont présentés par la figure 4. L'analyse de cette Figure montre globalement que dans la région de Ziguinchor (Figure 4A), la classe de grainage (200 à 210 noix/Kg) est la plus représentée soit $22,7 \%$ des échantillons analysés; suivi de la catégorie 230 à 240 noix/Kg, qui représente 18,2\% des échantillons. Les catégories excellente, Très bien, Très moyen et Juste acceptable représentent chacune 13,6 \%. Dans la Région de Sédhiou (Figure 4B), la classe (200 à 210 noix/Kg) représentant la qualité «moyenne », soit $22 \%$ de l'échantillon, est la plus dominante. Les deux classes qui représentent les qualités « excellentes » et « très bon » occupent respectivement 14,6\% et 12,2\%. La qualité (230 à 240 noix/Kg) représente quant à elle 14,6\%. Au niveau de la Région de Kolda (Figure 4C), c'est plutôt la classe (220 à 230 noix $/ \mathrm{Kg}$ ) qui domine soit $50 \%$ des échantillons analysés, représentant la qualité « juste acceptable ». L'absence de la catégorie « excellente » et « très bonne » est notée dans cette Région.
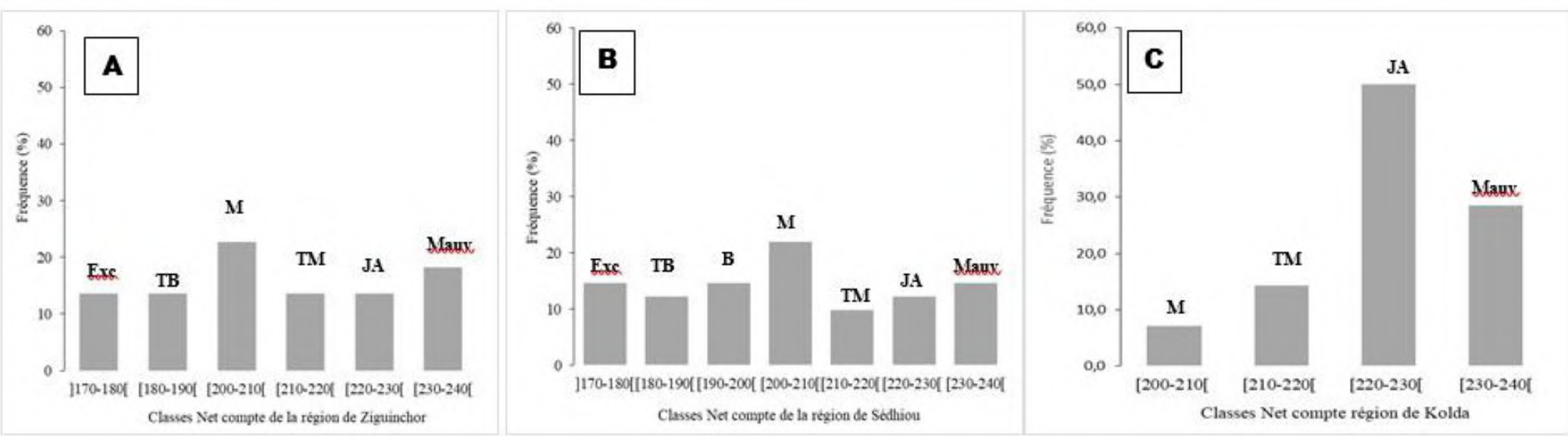

Figure 4 : La qualité du Grainage en fonction des $\mathbf{A}=$ Grainage Région de Ziguinchor $; \mathbf{B}=$ Grainage Région de Sédhiou $; \mathbf{C}=$ Grainage Région de Kolda)

$\mathbf{E x c}=$ Excellent, $\mathbf{T B}=$ Très Bon, $\mathbf{B}=$ Bon, Moy = Moyen, $\mathbf{T M}=$ Très Moyen, $\mathbf{J A}=$ Juste Acceptable, $\mathbf{M a u v}=$ Mauvais 


\section{L'analyse du taux de défaut dans les Régions de Ziguinchor, Sédhiou et Kolda}

Les résultats du taux de défaut sont présentés par la figure 5. Le taux de défaut varie d'une région à une autre. Ainsi dans la Région de Ziguinchor (Figure 5A), la classe (5-10\%) correspondant à la catégorie bonne représente 13,6 de l'échantillon, la catégorie dite mauvaise de classe (15-20\%) correspond à 22,7\% des noix et pour la catégorie bonne, (5$10 \%$ ), elle correspond à 63,6\%. Contrairement à la Région de Ziguinchor, dans la Région de Sédhiou (Figure 5B), le taux de défaut compris entre 5 et $10 \%$ représente 73,2\% des noix collectées suivi de la classe (10 à 15) avec 12,2\% et la classe de la catégorie mauvaise (15-20 \%) avec 14,6 \%.Les mêmes tendances sont observées dans la Région de Kolda (Figure 5C). Dans cette zone, la catégorie dite «Bonne » (5-10\%) représente $50 \%$, la classe (10-15\%) avec 21,4\% et la classe (15-20\%) correspondant à la catégorie « Acceptable » avec 28,6\%.
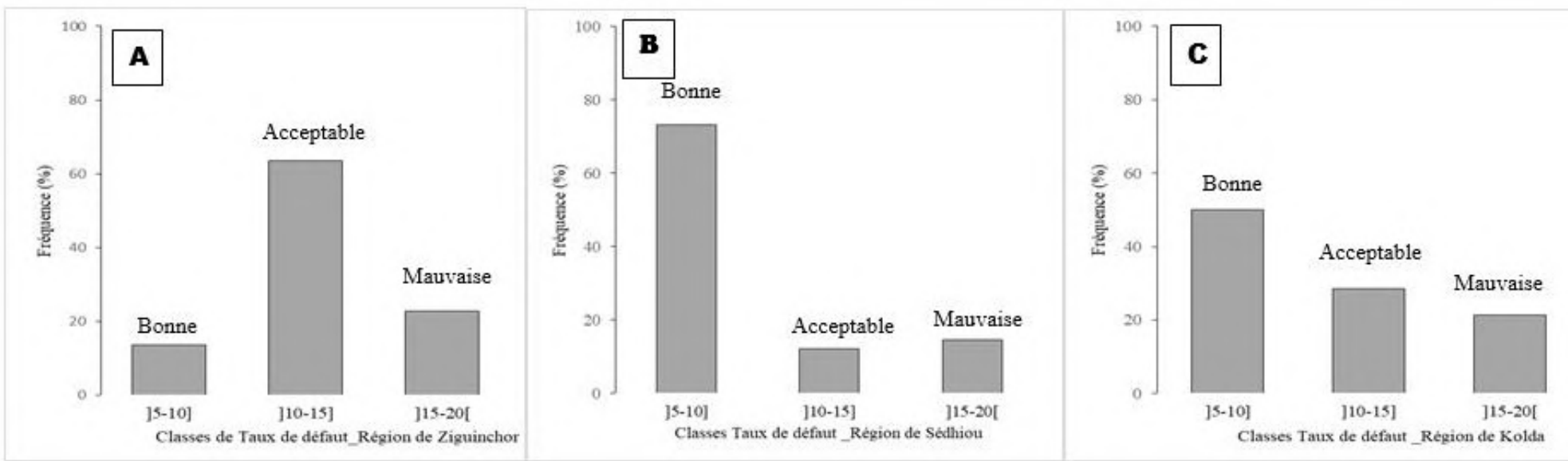

Figure 5 : Le taux de défaut des noix en fonction des Régions $(\mathrm{A}=$ Taux de défaut de la région de Ziguinchor $; \mathrm{B}=$ Taux de défaut de région de Sédhiou ; $\mathrm{C}=$ Taux de défaut de la région de Kolda 
Evaluation des rendements en amandes des différentes noix suivant les départements

Les rendements en amande varient d'un département à un autre. L'analyse de la variance entre les départements et les rendements en amande montre une différence significative $(\mathrm{p}=0,00399)$ entre Bounkiling et Médina Yoro Foula. Les rendements moyens par département sont de 26,43\% à Bignona, 28,34 \% à Bounkiling, 25,57 \% à Goudomp, 24,53 \% à Kolda, 23,57 $\%$ à Médina Yoro Foula, 26,42 \% à Oussouye, 27,03 \% à Sédhiou, 24,92 \% à Vélingara et $27,12 \%$ à Ziguinchor avec un coefficient de variation $(\mathrm{CV})$ de $8,33 \%$ (Figure 6). Toutefois dans tous les départements, les rendements sont acceptables par rapport à la norme indiquée qui doit être inférieur à $15 \%$, audessous de laquelle les noix sont rejetées.

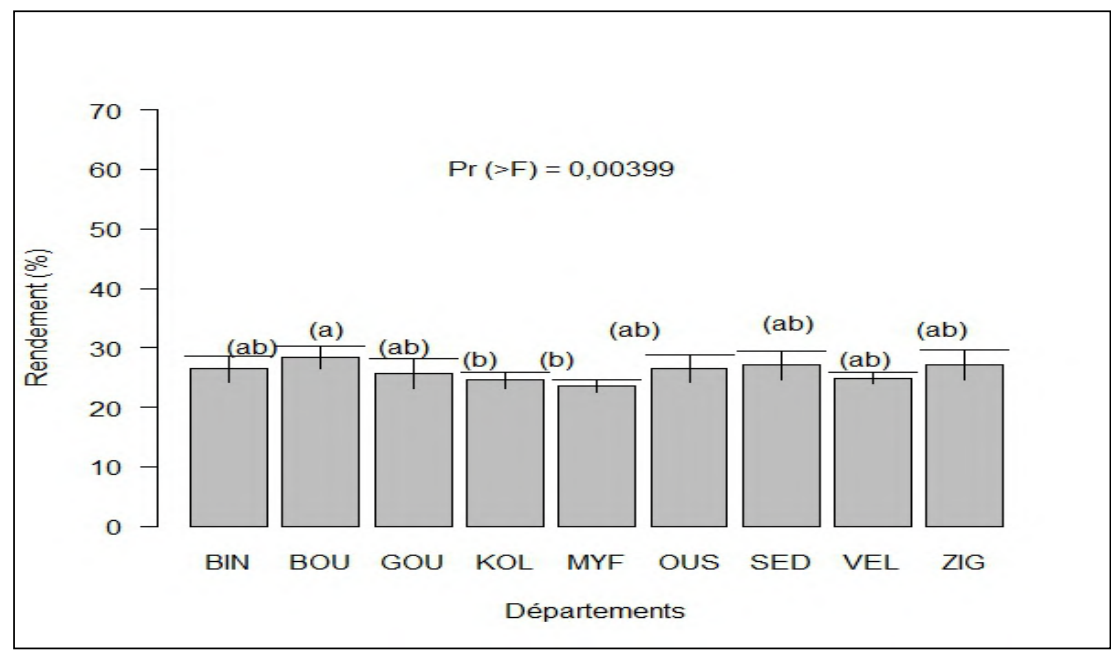

Figure 6 : Rendement en amande au niveau des différents départements BIN=Bignona, BOU=Bounkiling, GOU=Goudomp, KOL=Kolda, MYF=Médina Yoro Foula, OUS=Oussouye, SED=Sédhiou, VEL=Vélingara, ZIG= Ziguinchor

\section{Evaluation de la quantité d'amande utile (AU) contenu dans chaque échantillon de noix suivant les départements}

La quantité d'amande utile (AU) permet de renseigner sur la quantité d'amande transformable contenue dans un kilogramme de noix. Suivant les zones de provenance, l'analyse de la variance montre une différence significative $(\mathrm{p}=0,00399)$. Le test de comparaison des moyennes montre que le département de Bounkiling présente la moyenne en amande utile la plus élevée (283,4 g. $\left.\mathrm{kg}^{-1}\right)$ suivi de Ziguinchor (271,2 g. $\left.\mathrm{kg}^{-1}\right)$, de Sédhiou (270,35 g. $\left.\mathrm{kg}^{-1}\right)$, de Bignona

g.kg $\left.{ }^{-1}\right)$, de Kolda $\left(245,37\right.$ g. $\left.\mathrm{kg}^{-1}\right)$ et Médina Yoro Foula $(235,7$ g.kg-1) (Figure 7). 


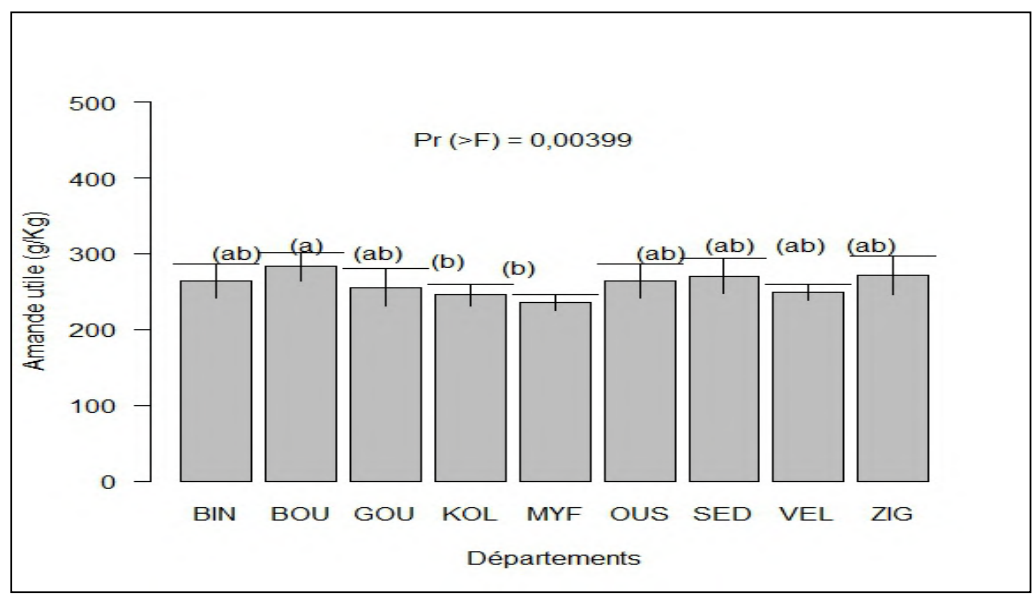

Figure 7 : Variation et en amande utile au niveau des différents départements BIN=Bignona, BOU=Bounkiling, GOU=Goudomp, KOL=Kolda, MYF=Médina Yoro Foula, OUS=Oussouye, SED=Sédhiou, VEL=Vélingara, ZIG= Ziguinchor 


\section{Analyse du KOR au niveau des Régions de Ziguinchor, Sédhiou et Kolda}

L'analyse montre que globalement sur l'ensemble des échantillons de la Région de Ziguinchor (Figure 8A), la qualité du KOR la plus représentée se trouve dans les classes (50-52 lbs) représentant la catégorie « Très bonne qualité » avec 72 $\%$ des noix analysées contre 9,1\% représentant la qualité «Qualité extrêmement mauvaise ». Les autres catégories (Qualité extrêmement mauvaise, Très mauvaise qualité, Qualité moyenne et Bonne qualité) sont évaluées à moins de $5 \%$ sur l'ensemble des échantillons. Dans la Région de Sédhiou (Figure 8B), le KOR de catégorie «Très bonne qualité » (50-52 lbs) est plus représentée soit 78,6\%. Les autres qualités sont évaluées à moins de $10 \%$.

A la différence de la Région de Ziguinchor et Sédhiou, dans la Région de Kolda (Figure 8C), c'est plutôt la catégorie «Bonne qualité » de KOR compris entre 48 et $50 \mathrm{lbs}$, qui est plus représenté soit 62,1\% suivi de la catégorie « Mauvaise qualité » avec 20,9\% des échantillons analysés.

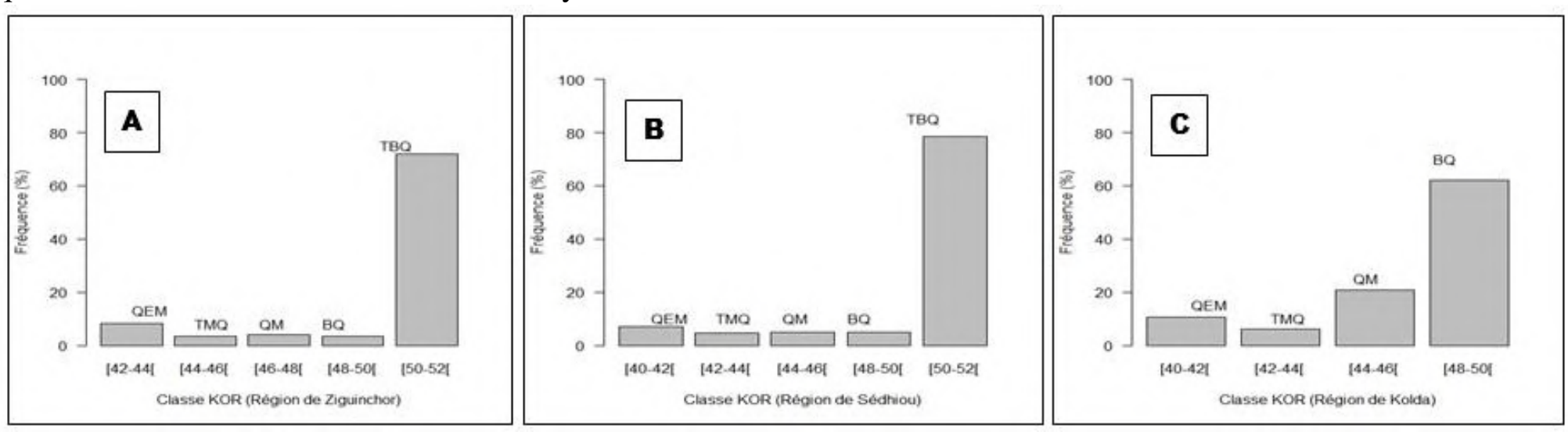

Figure 8 : La qualité du KOR en fonction des Régions ( $\mathrm{A}=$ KOR Région de Ziguinchor ; B = KOR Région de Sédhiou ; $\mathrm{C}=\mathrm{KOR}$ Région de Kolda)

$\mathrm{QEM}=$ Qualité Extrêmement Mauvaise $; \mathrm{TMQ}=$ Très Mauvaise Qualité $; \mathrm{MQ}=$ Qualité Moyenne $; \mathrm{BQ}=\mathrm{Bonne}$ Qualité ; TBQ = Très Bonne Qualité 


\section{Analyse comparative de grainage, taux de défaut et du KOR entre les départements de chaque région (Ziguinchor, Sédhiou et Kolda)}

Les résultats de l'analyse comparative de grainage, du taux de défaut et du KOR entre les départements de la région de Ziguinchor sont consignés dans le tableau 3. L'analyse de ce tableau montre que le meilleur grainage est obtenu dans le département de Ziguinchor (204 noix. $\mathrm{Kg}^{-1}$ ) suivi de Oussouye (208 noix. $\mathrm{Kg}^{-1}$ ) et Bignona (215 noix. $\left.\mathrm{Kg}^{-1}\right)$. Cependant l'analyse de la variance du grainage entre ces trois départements ne montre aucune différence significative $(\mathrm{p}=0,802)$.

Les meilleurs taux de défaut sont obtenus dans les départements de Ziguinchor et Oussouye respectivement $10 \%$ et $11 \%$. Toutefois, la différence n'est pas significative $(\mathrm{p}=0,084)$ entre les départements.

Par rapport à l'Out-Turn, les meilleures qualités de noix se situent dans les départements de Ziguinchor et Bignona avec des valeurs moyennes respectives de 50,2 lbs et 50,7 lbs (Très bonne qualité). Cependant, aucune différence n'a été notée ( $\mathrm{p}=0,814)$ entre les départements (Tableau 3).

Tableau 3 : Evaluation du grainage, du taux de défaut et l'Out-Turn entre les départements de la région de Ziguinchor

\begin{tabular}{|c|c|c|c|c|c|c|c|c|c|c|c|c|c|c|c|c|}
\hline Région & Département & \multicolumn{5}{|c|}{ Grainage } & \multicolumn{5}{|c|}{ Taux de défaut } & \multicolumn{5}{|c|}{ Out-Turn } \\
\hline \multirow{4}{*}{ Ziguinchor } & & STD & $\mathbf{R}$ & Min & Max & Moyenne & STD & $\mathbf{R}$ & Min & Max & Moyenne & STD & $\mathbf{R}$ & Min & Max & Moyenne \\
\hline & Ziguinchor & 29,77 & 14 & 182 & 270 & 208 & 3,3 & 14 & 8,4 & 15,3 & 10 & 3,28 & 14 & 40,6 & 51,8 & 50,2 \\
\hline & Bignona & 32,63 & 14 & 179 & 260 & 204 & 5,1 & 14 & 6,3 & 20 & 14 & 4,07 & 14 & 40,8 & 52 & 50,7 \\
\hline & Oussouye & 19,46 & 9 & 180 & 235 & 215 & 4,7 & 9 & 10,3 & 15 & 11 & 4,46 & 9 & 40,8 & 50,1 & 48 \\
\hline
\end{tabular}

Tableau 4 : Evaluation du grainage, du taux de défaut et l'Out-Turn entre les départements de la région de Sédhiou

\begin{tabular}{|c|c|c|c|c|c|c|c|c|c|c|c|c|c|c|c|c|}
\hline Région & Département & \multicolumn{5}{|c|}{ Grainage } & \multicolumn{5}{|c|}{ Taux de défaut } & \multicolumn{5}{|c|}{ Out-Turn } \\
\hline \multirow{4}{*}{ Sédhiou } & & STD & $\mathbf{R}$ & Min & Max & Moyenne & STD & $\mathbf{R}$ & Min & Max & Moyenne & STD & $\mathbf{R}$ & Min & Max & Moyenne \\
\hline & Sédhiou & 21,17 & 10 & 179 & 239 & 211 & 5,1 & 10 & 7,2 & 28,4 & 10,5 & 4,07 & 10 & 40 & 50,3 & 49,7 \\
\hline & Goudomp & 19,19 & 21 & 180 & 238 & 210 & 4,7 & 21 & 6 & 25,3 & 8,7 & 4,46 & 21 & 40 & 50,9 & 50,9 \\
\hline & Bounkiling & 11,98 & 10 & 190 & 233 & 215 & 3,3 & 10 & 5,1 & 15 & 12 & 3,28 & 10 & 41 & 50 & 48 \\
\hline
\end{tabular}


Les résultats de la qualité des noix au niveau des départements de la région de Sédhiou sont présentés dans le Tableau 4. L'analyse de la variance du grainage entre ces trois départements ne montre aucune différence significative $(p=0,07)$. Cependant les valeurs du grainage dans les départements sont de 210 noix. $\mathrm{Kg}^{-1}$ à Goudomp, 211 noix. $\mathrm{Kg}^{-1}$ à Sédhiou et 215 noix. $\mathrm{Kg}^{-1}$ à Bounkiling.

Le taux de défaut est de 8,7\% à Goudomp, $10,5 \%$ à Sédhiou et $12 \%$ à Bounkiling. Toutefois, la différence n'est pas significative $(p=0,485)$ entre les départements.

Par rapport à l'Out-Turn, la meilleure qualité de noix se situe dans le département de Goudomp (50,9 lbs) suivi de Sédhiou (49,7 lbs) et Bounkiling (48 lbs). Une différence a été notée entre les départements $(\mathrm{p}=0,0485)$ (Tableau 4).

La qualité des noix au niveau des départements de la région de Kolda est consignée dans le tableau 5. Ainsi le grainage moyen varie de 214 noix. $\mathrm{Kg}^{-}$ ${ }^{1}$ à Kolda, 225 noix. $\mathrm{Kg}^{-1}$ à Vélingara et 231 noix. $\mathrm{Kg}^{-1}$ à Médina Yoro Foula. Cependant l'analyse de la variance du grainage entre ces trois départements montre une différence significative $(p=0,0113)$.

Le taux de défaut le plus faible est obtenu dans le département de Vélingara (11,75\%) contre 12,7 \% à Koda et 12,24 \% à Médina Yoro Foula. Toutefois, la différence n'est pas significative $(p=0,904)$ entre les départements.

Par rapport à l'Out-Turn, la meilleure qualité de noix se situe dans le département de Kolda (49 lbs) suivi de Vélingara (47 lbs) et Médina Yoro Foula (46 Ibs). Aucune différence significative n'a été notée entre les départements $(\mathrm{p}=0,185)$ (Tableau 5). 
Tableau 5: Evaluation du grainage, du taux de défaut et l'Out-Turn entre les départements de la région de Kolda

\begin{tabular}{|c|c|c|c|c|c|c|c|c|c|c|c|c|c|c|c|c|}
\hline Région & Département & \multicolumn{5}{|c|}{ Grainage } & \multicolumn{5}{|c|}{ Taux de défaut } & \multicolumn{5}{|c|}{ Out-Turn } \\
\hline \multirow{3}{*}{ Sédhiou } & & $\begin{array}{c}\text { ST } \\
\text { D }\end{array}$ & $\mathbf{R}$ & Min & Max & $\begin{array}{c}\text { Moyen } \\
\text { ne }\end{array}$ & STD & $\mathbf{R}$ & Min & Max & $\begin{array}{c}\text { Moyen } \\
\text { ne }\end{array}$ & STD & $\mathbf{R}$ & Min & Max & $\begin{array}{c}\text { Moyen } \\
\text { ne }\end{array}$ \\
\hline & Kolda & 8,22 & 10 & 204 & 224 & 214 & 2,77 & 10 & 9,8 & 15,8 & 12,7 & 2,36 & 10 & 40,4 & 50 & 49 \\
\hline & $\begin{array}{ll}\text { Médina } & \text { Yoro } \\
\text { Foula } & \end{array}$ & 8,64 & 10 & 220 & 244 & 231 & 3,88 & 10 & 7,3 & 15 & 12,24 & 1,79 & 10 & 40 & 49 & 46 \\
\hline
\end{tabular}

Tableau 6 : Evaluation du grainage, du taux de défaut et l'Out-Turn entre les régions de Ziguinchor, Sédhiou et Kolda

\begin{tabular}{|c|c|c|c|c|c|c|c|c|c|c|c|c|c|c|c|}
\hline & \multicolumn{5}{|c|}{ Grainage } & \multicolumn{5}{|c|}{ Taux de défaut } & \multicolumn{5}{|c|}{ Out-Turn } \\
\hline Régions & STD & $\mathbf{R}$ & Min & Max & $\begin{array}{c}\text { Moyen } \\
\text { ne }\end{array}$ & STD & $\mathbf{R}$ & Min & Max & $\begin{array}{c}\text { Moyen } \\
\text { ne }\end{array}$ & STD & $\mathbf{R}$ & Min & Max & $\begin{array}{c}\text { Moyen } \\
\text { ne }\end{array}$ \\
\hline Ziguinchor & $\begin{array}{c}28,8 \\
7 \\
\end{array}$ & 22 & 157 & 270 & 208 & 5,29 & 22 & 7,3 & 15,8 & 12,94 & 2,1 & 22 & 40,6 & 52 & 50,7 \\
\hline Sédhiou & $\begin{array}{c}19,1 \\
1\end{array}$ & 41 & 175 & 258 & 207 & 4,53 & 41 & 5,1 & 28,5 & 13,72 & 4,5 & 41 & 40,3 & 52 & 50,9 \\
\hline Kolda & 9,91 & 14 & 204 & 244 & 224 & 2,92 & 14 & 6,3 & 25,9 & 12,26 & 3,96 & 14 & 40 & 50 & 49 \\
\hline
\end{tabular}


Analyse comparative de grainage, taux de défaut et du KOR au niveau des régions de Ziguinchor, Sédhiou et Kolda

Les résultats la qualité des noix au niveau de chaque région sont consignés dans le tableau 6. Ainsi les meilleurs grainages sont obtenus dans les régions de Ziguinchor (208 noix. $\mathrm{Kg}^{-1}$ ), suivi de Sédhiou (207 noix. $\mathrm{Kg}^{-1}$ ) et Kolda (224 noix. $\mathrm{Kg}^{-1}$ ). Cependant l'analyse de la variance du grainage entre ces trois régions montre une différence significative $(\mathrm{p}=0,0291)$.

Les taux de défaut sont de 12,94\% à Ziguinchor, 12,26 \% à Kolda et $13,72 \%$ à Sédhiou. Toutefois, la différence n'est pas significative $(p=0,55)$ entre les régions.

Par rapport à 1'Out-Turn, les valeurs moyennes par région sont de 50,7 lbs à Ziguinchor, 50,9 lbs à Sédhiou et $49 \mathrm{lbs}$ à Kolda. Une différence significative a été notée entre les régions $(\mathrm{p}=0,00461)$ (Tableau 6).

\section{Discussion}

Dans les différentes régions, le grainage varie de 204 à 214 noix. $\mathrm{kg}^{-1}$ à Ziguinchor, de 201 à 215 noix. $\mathrm{kg}^{-1}$ à Sédhiou et de 212 à 232 noix. $\mathrm{kg}^{-1}$ à Kolda. Ces valeurs du grainage sont dans la fourchette de la norme définie par la CEDAO (200 à 250 noix. $\mathrm{kg}^{-1}$ ) mais un peu en dessous de celle de la norme ISO comprise entre 180 et 200 noix. $\mathrm{kg}^{-1}$ (Lautié et al, 2001). Toutefois, le Bénin avec 100 à 200 noix. $\mathrm{kg}^{-1}$, a le meilleur grainage comparé à celui du Sénégal (iCA, 2011).

Le KOR moyen est de 50,7 lbs pour la région de Ziguinchor, 50,9 lbs pour Sédhiou et $49 \mathrm{lbs}$ pour celle de Kolda. Avec l'introduction de la norme ISO 6477 en 1988 pour harmoniser et fournir et schéma de contrôle de la qualité (ONU, 2002), les noix brutes de cajou ont fait l'objet d'une norme conjointe entre les Etats membres de CEE et ceux de l'ONU qui fixe les normes de conditions minimales qu'une noix doit remplir pour être vendue sur le marché international. Selon ladite norme, un bon KOR doit être supérieur à $50 \mathrm{lbs}$. Ces résultats obtenus sur le KOR dans le cadre de cette étude sont supérieurs à ceux obtenus au Bénin, la Côte d'Ivoire et le Nigéria. En effet, Pal (2016) montre que le KOR moyen du Bénin, de la Côte d'Ivoire et celui du Nigéria varie respectivement 47,8 lbs, 47,6 lbs et 45,3 lbs. La Guinée Bissau, l'Inde et le Vietnam ont les meilleurs KOR qui varient respectivement $52,5 \mathrm{lbs}, 52,3 \mathrm{lbs}$ et $52,3 \mathrm{lbs}$. C'est un indicateur très important dans le commerce international des noix brutes. Il conditionne ainsi le prix du marché mondial. A ce titre, l'Inde, le Vietnam, la Guinée-Bissau et la Tanzanie perçoivent en moyenne des prix, $25 \%$ plus intéressants que ceux leurs pairs asiatiques et africains (Pal, 2016). Le bon KOR des noix brutes du Sénégal pourrait être due à la diversité des variétés améliorées introduites et aux conditions environnementales très favorables pour le développement de l'espèce. La très bonne qualité des noix de la Guinée Bissau, pays voisin de 
la Casamance confirmerait cette hypothèse. Selon ISRA (2013), onze (11) provenances ont été introduites dans la région de Fatick, onze (11) dans la région de Kolda, douze (12) dans la région de Sédhiou et onze (11) dans la région de Ziguinchor. Dans ces différentes zones de production, les variétés locales restent dominantes au niveau des plantations (Ndiaye et al., 2017). Les appuis de certains projets comme le PASA (dans la région de Fatick), le projet forestier et le PADEC (au Sud) ont favorisé l'introduction de semences améliorées. Le KOR relativement bas des noix provenant des départements la région de Kolda (46-49 lbs) pourrait être dû aux variétés introduites dans cette zone qui donnent des noix de petits calibres comparé aux six (6) autres départements. Selon Touré et al. (2018), les variétés locales dites « Henry et James » seraient les plus dominantes dans la région de Kolda, caractérisées par des noix de petite taille.

Le taux de défaut est le paramètre qui conditionne la qualité des noix brutes. Il pourrait être influencé par le taux d'humidité, les moisissures et le mode de stockage ou de conditionnement des noix après la récolte. Cette étude a montré qu'il varie en moyenne de 7,5 à 10,6\% suivant les neuf (9) départements. Ce qui dépasse la norme UEMOA qui est de $8 \%$ (Arlène et Broutin, 2009). Ces taux sont également en dessous de ceux du Bénin qui est 14,07\% (iCA, 2011). La valeur relativement élevée des amandes défectueuses serait due à la récolte précoce des noix, au manque de matériel et d'unité de stockage par les producteurs. C'est pourquoi, la région de Ziguinchor et Sédhiou disposant d'un KOR moyen 50 lbs ont enregistré une baisse de leur qualité (40-45 lbs) en 2017 (Ricau, 2013). Par ailleurs, la période de récolte pourrait influencer la qualité des noix. En effet, les noix récoltées avant la saison des pluies présentent les meilleures qualités comparées à celles obtenues pendant l'hivernage qui sont plus exposées à l'humidité.

\section{Conclusion}

Cette étude a permis de monter que la qualité des noix brutes varie d'une région à une autre. Ainsi les noix présentant un bon KOR et un taux de défaut bas sont rencontrées dans les régions de Ziguinchor et Sédhiou. Globalement le KOR moyen la Casamance est de 50,2 lbs, le taux de défaut moyen est 12,92 $\%$ et le grainage moyen est de 213 noix/Kg. Toutefois la Casamance, malgré les contraintes de la filière dispose d'un bon KOR, un atout qui valoriserait les noix à l'exportation. Cependant des efforts considérables sont à fournir allant dans le sens de booster la production à travers l'introduction des variétés productives et adaptées à la zone agroécologique de la Casamance. L'encadrement des producteurs dans la conservation et le conditionnement des noix permettrait de diminuer le taux de défaut et de donner plus de valeur aux noix pour la commercialisation. 


\section{Remerciement}

Les auteurs expriment leur gratitude au ministère de l'enseignement supérieur de la recherche et de l'innovation du Sénégal à travers le Fonds d'Impulsion pour la Recherche Scientifique et Technique (FIRST 2015) qui a permis la réalisation de cette étude.

\section{References:}

1. Aina, M. M. S. (1996). L'anacardier dans le système de production au niveau paysan: une approche de rentabilité économique et de la gestion du terroir dans la commune rurale d'Agoua (Zou). Thèse d'ingénieur agronome, FSA, UNB. $112 \mathrm{p}$

2. ANSD. (2011). Sénégal et ICF International. Enquête Démographique et de Santé à Indicateurs Multiples au Sénégal (EDSMICS) 2010-2011. Calverton, Maryland, USA : ANSD et ICF International. $520 \mathrm{p}$

3. ANSD. (2015). Sénégal : Enquête Démographique et de Santé Continue (EDS - Continue). $270 \mathrm{p}$

4. Arlène A et Broutin C. (2009). Normes de qualité pour les produits agroalimentaires en Afrique de l'Ouest. Agence française de développement, département de recherche. $230 \mathrm{p}$

5. Ba M. (2018). Suivi de la productivité des anacardiers géoréférenciés dans le Balantacounda. Rapport de mission. PADEC/Sédhiou. $6 \mathrm{p}$

6. Camara B. (2018). Caractérisation agro-écologique et socioéconomique des parcs à Elaeis guineensis Jacq. Et Faidherbia albida (Del.) Chev. et leurs influences sur la productivité du riz pluvial en Basse Casamance (Sénégal). Thèse de doctorat, Université Assane Seck de Ziguinchor.140 p

7. Core-team. (2018). R : une langue et un environnement pour les statistiques, l'informatique. Fondation pour l'information statistique. Vienne, Autriche. URL = https://www.R-project.org consulté le 14 mars 2019.

8. Dandjinou B. (2016). Global Competitiveness : the Real Issues and How to Adress It. Festival et Expo mondiaux 2016 sur le cajou organisés par l'ACA. Guinea-Bissau, 19-22 septembre 2016. 25 p

9. DDP. (2002). Norme de CEE-ONU DDP-01. Concernant la commercialisation et le contrôle de la qualité commerciale des noix en coque. Edition 2002. $11 \mathrm{p}$

10. FAOSTAT. (2019). Crops Cashews nuts, with shell. [Base de données] (consulté le 5 janvier 2020).

11. Gagnon, B. M. (1998). Etude de la filière anacarde au Bénin. Programme "Entreprenariat Bénin" Bénin. Rapport définitif. 87 p 
12. Gaye A. T. Lô H. M., Djimbira S. S., Fall M. S. et Ndiaye I. (2015). Sénégal: revue du contexte socioéconomique, politique et environnemental. Rapport d'étude. $88 p$

13. Hassine, N. B. et Matoussi M. S. (2006). Compétitivité et qualité des produits: Le cas de

l'agriculture et de l'agroalimentaire. $36 \mathrm{p}$

14. Hien S. (2019). N'kalô. Aperçu de l'évolution de la production d'anacarde et évolution du marché de noix brutes de cajou dans la sous-région et perspectives pour 2019/2020. Forum sur le cajou sahélien. Du 05 au 07 août 2019 à Bamako (Mali). 16 p

15. iCA (initiative pour le cajou africain). (2014). African Cashew Alliance. Promouvoir les noix de cajou d'Afrique dans le monde entier. Bulletin mai $2014 n^{\circ} 4$. ISSN $2458-7362.15 \mathrm{p}$

16. iCA. (2011). Cartographie du KOR pour la campagne 2011 au Bénin. Initiative de Cajou Africain Bénin: Fiche de Résultats nº03/2011.2p

17. ISRA. (2013). Caractérisation des variétés d'anacardes au Sénégal et en Gambie. Sommaire pour les pépiniéristes de CEP2. $21 \mathrm{p}$

18. Lautié E., Dorniera M., Filhoc M., De-Souza F et Reynes M. (2001). Les produits de l'anacardier: caractéristiques, voies de valorisation et marchés. Fruits, 2001, 56, 235-248

19. Ndiaye S. (2014). Caractérisation des plantations à base d'anacardier dans la Communauté rurale de Djibanar. Mémoire de Master. Université Assane Seck de Ziguinchor. $58 \mathrm{p}$

20. Ndiaye S., Charahabil M. M.et Diatta M. (2017). Caractérisation des Plantations à Base d'anacardier dans les communes de Kaour, Goudomp et Djibanar. European Scientific Journal April 2017 edition Vol.13, No.12. 242-257. ISSN: 1857 - 7881 (Print) e - ISSN 8577431. doi: 10.19044/esj.2017.v13n12p242

21. ONU. (2002). Norme CEE-ONU DDP-01concernant la commercialisation et contrôle de la qualité des noix en coque. Groupe de travail des normes de qualité des produits agricoles à sa 59ème session. Document TRADE/WP.7/2002/9/Add.14, $11 \mathrm{p}$

22. PADEC. (2016). Programme de l'Appui au Développement Economique de la Casamance. Enquête sur le sous-secteur de l'anacarde au Sénégal. Résumé global. $23 \mathrm{p}$

23. Pal S. (2016). Compétitivité mondiale : Données de référence sur l'industrie mondiale de transformation du cajou. Festival et Expo mondiaux 2016 sur le cajou organisés par l'ACA. Guinea-Bissau, 1922 septembre 2016. $15 \mathrm{p}$

24. Rakotomala R. (2011). Test de normalité : techniques empiriques et tests statistiques. Version 2. Université Lumière Lyon 2. $59 \mathrm{p}$ 
25. Ricau P. (2013). Connaître et comprendre le marché international de l'anacarde. Rongead- N'kalô. www.rongead.org 49 p

26. Rongead. (2015). L'out-turn ou comment mesurer la qualité de l'anacarde? Projet de professionnalisation de la filière anacarde, mise en œuvre IFCI/Rongead. 36p

27. Sagna P. (2007). Caractéristiques climatiques, Atlas du Sénégal, Paris, Les Editions Jeune Afrique, 66-69.

28. Sampat P. N. (2015). Cashew Price Analysis - 2005 to 2014. Cashew Market Report - April 5, 2015. $2 \mathrm{p}$

29. Sène A. M. (2019). Agrobusiness de l'anacarde en Casamance (Sénégal) : atouts, contraintes et perspectives d'industrialisation. European Scientific Journal May 2019 édition 15 (15) : 363-377.

30. Seters J. V. et Konnon D. (2018). Capitalisation des expériences et acquis de la Côte d'Ivoire en matière de politiques publiques, de structuration et de gestion de la filière anacarde. Ecdpm Document de réflexion $\mathrm{n}^{\circ} 234.56 \mathrm{p}$

31. Shaffer J. (2007). Controlling the false discovery rate with constraints: The Newman-Keuls test revisited. Biometrical Journal. 49: 136-143.

32. Touré M. A., Faye E., Malou G., Diatta M., Ndiaye Samba S. A. et Gassama Y. K. (2018). Traits morphométriques et germination des noix de Anacarde occidentale L. au Sénégal. Afrique SCIENCE 14(2) (2018) 215 - 226. ISSN 1813-548X, http://www.afriquescience.info 